\title{
Partner-Specific Sexual Behaviors Among Persons with Both Main and Other Partners
}

\author{
By Amy Lansky, James C. Thomas and Jo Anne Earp
}

Context: If men and women engage in different sexual behavior with main partners than with other types of partners, then programs aimed at preventing the spread of sexually transmitted diseases (STDs) may need to address individuals' differential risk with each partner type.

Methods: Relationship characteristics, partner risk behaviors and sexual behaviors are examined among 123 male and 106 female STD clinic patients who had both main and other partners. Individual-level comparisons are made for two types of partner pairs: main vs. other frequent (side) partners and main vs. casual partners.

Results: Among men and women with both main and side partners, the proportion who had known only their main partner for at least a year (48\% of men and $41 \%$ of women) was significantly higher than the proportion who had known only their side partner for that long $(2 \%$ and $9 \%$, respectively); no other variable differed significantly by partner type. Among those with main and casual partners, both men and women were more likely to use alcohol or drugs before or during sex with main partners only (15\%) than with casual partners only (1-3\%). Women with main and casual partners were more likely to have oral sex only with main partners than only with casual partners (37\% vs. 3\%), and were more likely to use condoms only with casual partners than only with main partners (33\% vs. $4 \%$ ).

Conclusions: Providers need to ask individuals about their sexual behaviors with different partner types, and should tailor prevention messages to an individual's risks and reproductive intentions with each partner.

Family Planning Perspectives, 1998, 30(2):93-96

$\mathrm{M}$ ore than 600,000 Americans have developed AIDS since the beginning of the epidemic, and many of them contracted HIV through sexual contact with an infected partner. The proportion of infected women who acquired the virus through sexual activity with an infected man has risen steadily, and in 1996, it exceeded the proportion infected through injection-drug use ( $40 \%$ vs. $35 \%$ ). Among men, sexual contact with an infected male has been the most common mode of transmission throughout the epidemic, accounting for $59 \%$ of infections. ${ }^{1}$ Given the importance of sexual contact in the spread of HIV among both men and women, it is imperative to understand the variability of sexual behaviors that put people at risk.

Having multiple sex partners may increase an individual's chances of coming into contact with a partner who is infected. However, it does not necessarily increase the risk of acquiring a sexually transmitted disease (STD), as consistent and correct use of condoms can lower the probability of transmission of HIV and other STDs. ${ }^{2}$ In addition, the prevalence of STDs varies within the population, ${ }^{3}$ so an individual's choice of partners will also affect the probability of infection.

Definitions and labels for types of sex partners vary from study to study. Most commonly found is a dichotomy between "main" partners (also called "regular," "steady" or "primary" partners) and "other" partners (also called "casual" or "secondary" partners). Some studies make further distinctions for paying partners or customers, anonymous partners ${ }^{4}$ or new partners, ${ }^{5}$ in one study, women described men with whom they had unprotected sex, or what they described as "enjoyable sex" or "affection," as "side dishes." 6 Given these different labels, partner type may best be understood as a marker for the dynamics operating in a relationship.

To make inferences about the sexual behavior of people with both main and other partners, the appropriate analysis is at the individual level ("within-subjects" analy- sis). Because individuals with more than one type of partner give responses for each type, researchers must use statistical tests that account for multiple responses from each individual.

The purpose of our study was to determine whether assumptions about differences in condom use and other sexual behaviors with main and other partners can be extrapolated, using within-subjects analysis, to the individual level. If partner type is, indeed, a marker for differences in dynamics within a sexual relationship, then such differences would indicate the necessity of providing tailored prevention programs that address an individual's differential risk with each partner type.

\section{Methods}

Study Setting and Sample

Data for this analysis came from the Sexually Transmitted Epidemic Prevention Project, a study of STD patients in a rural North Carolina county. This county was chosen for the project because of its extremely high rates of syphilis, gonorrhea and HIV. ${ }^{7}$ The project included a formative research phase, a survey of patients with initial and repeat infections attending the county public health STD clinic, and ongoing qualita-

Amy Lansky is epidemic intelligence service officer, National Center for HIV, STD, and TB Prevention, Division of HIV / AIDS Prevention, Centers for Disease Control and Prevention, Atlanta. Jo Anne Earp is professor and chair, Department of Health Behavior and Health Education; James C. Thomas is associate professor, Department of Epidemiology; and at the time of this study, Amy Lansky was a doctoral student, Department of Health Behavior and Health Education-all at the School of Public Health, University of North Carolina, Chapel Hill. This research was supported by grant U01-AI31496 from the National Institute of Allergy and Infectious Diseases and by National Research Service Award T32-HS00032 from the Agency for Health Care Policy Research to the Cecil G. Sheps Center for Health Services Research. The authors acknowledge Louis Latour and the staff of the health department in which the study was conducted for assistance in data collection, and Gary Koch for consultation on statistical methods. 
Table 1. Percentage of sexually transmitted diseases clinic patients with multiple partner types, by selected characteristics, rural North Carolina, 1992-1994

\begin{tabular}{lll}
\hline Characteristic & $\begin{array}{l}\text { Women } \\
(\mathrm{N}=106)\end{array}$ & $\begin{array}{l}\text { Men } \\
(\mathrm{N}=123)\end{array}$ \\
\hline Black & 85 & 93 \\
High school graduate & $69^{\star}$ & 56 \\
Lives with partner & 20 & 19 \\
Has main partner & 95 & 93 \\
Has side partner & 48 & 50 \\
Has casual partner & 72 & 80 \\
>3 partners in past 3 months & $30^{\star * *}$ & 57 \\
Main partner has other partners & $76^{\star * *}$ & 44 \\
\hline
\end{tabular}

${ }^{\star} \mathrm{p}<.05$. ${ }^{\star \star \star} \mathrm{p}=.001$. Note: Education is adjusted for age.

tive data collection in the community. One objective of the project was to examine relationships with different types of partners.

All county residents visiting the clinic between August 1992 and January 1994 for a regular exam (i.e., other than to receive a test of cure, ongoing treatment, an HIV or pregnancy test only, or condoms) were eligible for the survey. Those agreeing to participate signed a consent form before the interview.

Only two men and no women reported same-sex partners; therefore, we restricted the analysis to persons with partners of the opposite sex in the past three months. We excluded respondents who reported no sex partners and, because of our interest in within-subjects analyses for those with multiple types of sex partners, those who reported only one type.

\section{Measures and Analyses}

The project's formative research phase provided background information about partner types and guided us in the wording of questions. We developed a 147-item structured questionnaire, which was administered by trained interviewers, and a shorter version for use when the interviewer did not have time to administer the full interview.*

Respondents were asked if they had a main partner, "like a husband or wife, or a boyfriend or girlfriend. Someone you see a lot." Those who reported having a main partner were asked: "After [name of main partner] is there anyone you have sex with more often than others?" The interviewers added the description "a second main squeeze" as part of the definition. If the respondent said yes, this partner was categorized as a "side partner." Thus, respondents themselves decided whether a second partner was a side or casual partner. Those who had no side partner or who had three or more partners were con-

*The analyses included in this article are based on data from the long form. sidered to have "casual" partners. (The terms "side" and "casual" partners were not used with respondents.)

Conceptually, we viewed side partners as being somewhere between main and casual partners, in terms of both frequency of intercourse and relationship status. In reality, these categories and definitions of partner types may not be mutually exclusive. However, the possible fluidity of partner types cannot be captured in our data, since our survey was cross-sectional.

Respondents who reported main or side partners were asked about characteristics of each relationship (e.g., how long they had known their partner) and about these partners' risk behaviors (e.g., whether they had partners other than the respondent). All respondents were asked whether, in the past three months, they had engaged in vaginal, anal or oral intercourse with each partner type and how frequently they had used condoms for each type of intercourse. (The three-month period was chosen to minimize recall bias.) We coded condom use as consistent if the respondent answered "every single time" or inconsistent if any other response was given. This coding scheme was chosen because consistent condom use is effective in preventing STDs.

Respondents were asked if they used alcohol or other drugs before or during sex, and were asked about whether and what kind of birth control they used with each partner (although they were not asked about the frequency with which they used birth control). Gender was coded by the interviewer, and other demographic variables (e.g., age and variables measuring socioeconomic status) were included in the questionnaire.

We used the chi-square test for demographic differences between men and women. For the within-subjects analyses of partner type differences, we used a statistical test for dependent data; $p$ values reported for differences by partner type are from the corrected McNemar's chi-square test. This test determines the probability that the proportion with the response of interest in one condition is greater than the proportion with the response of interest in the second condition (e.g., the probability that the proportion engaging in some behavior with their main partner but not their casual partner is greater than the proportion engaging in that behavior with their casual partner but not their main partner).

All within-subjects analyses are reported separately for women and men. We used EpiInfo 6.02 to calculate the corrected McNemar's test; all other data were analyzed using SAS 6.12.

\section{Results}

\section{Sample Characteristics}

Half of the 2,888 STD patients eligible for the study were asked to participtate, and virtually all of these (49\% of eligible patients) completed interviews. Patients who were interviewed did not differ from those not interviewed with regard to sex or age, but were more likely to be black ( $86 \%$ vs. $74 \%$ ). Of the patients surveyed, $47 \%$ were interviewed using the long form of the questionnaire. Those who responded to the short form did not differ from persons who completed the long form in terms of age, race, sex, number of partners or the proportion who had a main partner.

Of the 661 patients who completed the long form of the questionnaire, 35\% met the inclusion criteria for this analysis; these 123 men and 106 women constituted our final sample. The sample was predominantly black (93\% of men and $85 \%$ of women-Table 1). Women were significantly younger than men (median ages, 22 and 23 years, respectively-not shown) and were significantly more likely to have completed high school (69\% vs. 56\%). Only $19 \%$ of men and $20 \%$ of women lived with a partner at the time of the interview.

No significant differences were found between men and women in the proportions reporting different partner types. Men were more likely than women to have had three or more partners in the preceding three months (57\% vs. $30 \%$ ), but women were more likely than men to report that their main partner had other partners (76\% vs. $44 \%$ ). The practice of exchanging sex for money, drugs or other items was more common with casual partners (13\% of women and $7 \%$ of men) than with side partners (fewer than $5 \%$ of each).

For the within-subjects analyses, we report on 45 women and 54 men who had a main partner and a side partner, and 71 women and 90 men who had main and casual partners. No tests were conducted for differences among those with side and casual partners because of small sample size (20 women and 37 men). Included in both the main vs. side and the main vs. casual analyses are 10 women and 21 men who had all three partner types.

\section{Main vs. Side Partners}

Among respondents with both main and side partners, $41 \%$ of women and $48 \%$ of men had known their main partner, but not their side partner, for at least one year; by contrast, only $9 \%$ and $2 \%$, respectively, had known their side partner, but not their main partner, for that long (Table 2). No other relationship characteristics or 
any risk behaviors of the respondents' partners were significantly different according to partner type.

More than $95 \%$ of respondents engaged in vaginal sex, and fewer than $5 \%$ engaged in anal sex, with both partner types; consequently, there were no statistically significant differences by partner type for these behaviors among women and men. No significant differences in other behaviors were found when we compared main and side partners. The data suggest that more women used alcohol before or during sex with their main partner than with their side partner, but this difference was of borderline statistical significance $(\mathrm{p}=.08)$.

We also examined relationship characteristics and behaviors that respondents said applied to both their main and their side partners or to neither. The majority of women reported that both their main and their side partners had other partners $(58 \%)$, that they did not use condoms consistently for vaginal sex with either partner type $(75 \%)$ and that they used some type of birth control method with both partners (66\%); common methods used were oral contraceptives and tubal ligation, which do not vary by partner type because they are not dependent on the sexual act. Similarly, the majority of men said they did not use condoms consistently with either their main or their side partner $(77 \%)$, but that they used some contraceptive method with both $(57 \%)$.

\section{Main vs. Casual Partners}

For respondents reporting both main and casual partners, differences by partner type in the proportions engaging in vaginal and anal sex were not statistically significant (Table 3). The use of birth control also did not differ by partner type, for reasons noted previously.

Women were significantly more likely to engage in possibly risky behaviors only with main partners than only with casual partners: Whereas 37\% reported oral sex with their main but not their casual partner, only $3 \%$ engaged in this behavior with their casual but not their main partner; $15 \%$ reported using alcohol or drugs before or during sex with their main partner only, compared with 1-3\% with their casual partner only. At the same time, women were less likely to use condoms, a preventive behavior, only with their main partner $(4 \%)$ than only with their casual partner (33\%).

Men were more likely to use alcohol or drugs before or during sex with their main but not their casual partner (13-14\%) than with their casual but not their main partner $(3 \%)$. The data suggest that condom

Table 2. Percentage distribution of STD clinic patients with main and side partners, by partner to which measures of relationship and behavior apply, according to sex

\begin{tabular}{|c|c|c|c|c|c|c|c|c|c|}
\hline \multirow[t]{2}{*}{ Measure } & \multicolumn{4}{|c|}{ Women $(\mathrm{N}=45)$} & \multicolumn{4}{|c|}{ Men $(\mathrm{N}=54)$} & \multirow[t]{2}{*}{ Total } \\
\hline & $\begin{array}{l}\text { Main } \\
\text { only }\end{array}$ & $\begin{array}{l}\text { Side } \\
\text { only }\end{array}$ & Both & Neither & $\begin{array}{l}\text { Main } \\
\text { only }\end{array}$ & $\begin{array}{l}\text { Side } \\
\text { only }\end{array}$ & Both & Neither & \\
\hline \multicolumn{10}{|l|}{ Relationship characteristic } \\
\hline Known $>1$ year & 41 & $9^{* *}$ & 41 & 9 & 48 & $2^{\star *}$ & 24 & 26 & 100 \\
\hline Live in same county & 5 & 16 & 70 & 9 & 11 & 13 & 69 & 7 & 100 \\
\hline \multicolumn{10}{|l|}{ Partner's risk behavior } \\
\hline Other partners & 11 & 24 & 58 & 7 & 19 & 13 & 29 & 21 & 100 \\
\hline History of STD & 20 & 23 & 6 & 51 & 17 & 13 & 2 & 67 & 100 \\
\hline History of injection-drug use & 9 & 9 & 7 & 75 & 6 & 6 & 2 & 86 & 100 \\
\hline \multicolumn{10}{|l|}{ Sexual behavior } \\
\hline Vaginal sex & 0 & 2 & 98 & 0 & 2 & 0 & 98 & 0 & 100 \\
\hline Anal sex & 2 & 2 & 2 & 93 & 4 & 4 & 0 & 92 & 100 \\
\hline Oral sex & 27 & 11 & 18 & 44 & 22 & 9 & 22 & 46 & 100 \\
\hline Condom use $†$ & 7 & 18 & 0 & 75 & 9 & 9 & 4 & 77 & 100 \\
\hline Birth control use & 7 & 9 & 66 & 18 & 13 & 6 & 57 & 24 & 100 \\
\hline Alcohol use with sex & 16 & 2 & 34 & 48 & 7 & 4 & 52 & 37 & 100 \\
\hline Drug use with sex & 11 & 5 & 16 & 68 & 6 & 6 & 21 & 68 & 100 \\
\hline
\end{tabular}

${ }^{* \star} \mathrm{p}<.01$. FFor vaginal sex only. Note: $\mathrm{p}$ value is for McNemar's test, which considers discordant responses only.

use was more likely with casual partners, but the difference is only marginally statistically significant $(\mathrm{p}=.08)$.

\section{Discussion}

The findings of our analyses of main vs. casual partners are consistent with those of previous research showing that individuals use condoms more consistently for vaginal sex with other partners than with main partners. Similar results have been reported from samples of STD clinic patients, ${ }^{8}$ family planning clinic clients, ${ }^{9}$ women at high risk for HIV infection ${ }^{10}$ and college students, ${ }^{11}$ as well as from national surveys in the United States ${ }^{12}$ and in France. ${ }^{13}$

In a national survey of adult heterosexuals in the United States, ${ }^{14}$ 7\% of respondents with both primary and secondary partners used condoms consistently with secondary but not with primary partners; statistical tests of differences between primary and secondary partners were not reported. Black and Hispanic youth with multiple partners were found to engage in oral sex more frequently with a "main" (married or cohabiting) partner than with "well-known" (not married or cohabiting) or "casual" partners; consistent condom use was more likely with well-known partners than with main partners..$^{15}$ A study of heterosexual couples in California in which one partner was HIV-positive found that the consistency of condom use varied by type of partner. ${ }^{16}$ One explanation for partner-specific differences in condom use is that condom use may diminish over time in a relationship. ${ }^{17}$

Drug and alcohol use before or during sex are STD-related problems, for several reasons. There is a risk of HIV infection from injection-drug use, and the links between crack use, syphilis and HIV have been well documented. ${ }^{18}$ Alcohol use may be a marker for engaging in multiple risktaking behaviors, or it may affect judgment and decision-making regarding safer sex. ${ }^{19}$ High rates of alcohol use in rural areas underscore the need to provide education to persons living in these areas about decision-making regarding safer sex when under the influence of alcohol. Educational messages about safer sex should also address the intertwined issues of sub-

Table 3. Percentage distribution of STD clinic patients with main and casual partners, by partner to which measures of behavior apply, according to sex

\begin{tabular}{|c|c|c|c|c|c|c|c|c|c|}
\hline \multirow[t]{2}{*}{ Sexual behavior } & \multicolumn{4}{|c|}{ Women $(\mathrm{N}=71)$} & \multicolumn{4}{|c|}{ Men $(\mathrm{N}=90)$} & \multirow[t]{2}{*}{ Total } \\
\hline & $\begin{array}{l}\text { Main } \\
\text { only }\end{array}$ & $\begin{array}{l}\text { Casual } \\
\text { only }\end{array}$ & Both & Neither & $\begin{array}{l}\text { Main } \\
\text { only }\end{array}$ & $\begin{array}{l}\text { Casual } \\
\text { only }\end{array}$ & Both & Neither & \\
\hline Vaginal sex & 1 & 0 & 99 & 0 & 2 & 0 & 98 & 0 & 100 \\
\hline Anal sex & 7 & 0 & 0 & 93 & 0 & 0 & 1 & 99 & 100 \\
\hline Oral sex & 37 & $3^{\star \star}$ & 17 & 44 & 24 & 14 & 16 & 47 & 100 \\
\hline Condom use $†$ & 4 & $33^{\star \star}$ & 1 & 61 & 5 & 14 & 3 & 78 & 100 \\
\hline Birth control use & 4 & 13 & 63 & 20 & 18 & 11 & 52 & 18 & 100 \\
\hline Alcohol use with sex & 15 & $3^{*}$ & 45 & 37 & 13 & $3^{*}$ & 51 & 33 & 100 \\
\hline Drug use with sex & 15 & $1^{\star *}$ & 14 & 69 & 14 & $3^{*}$ & 14 & 69 & 100 \\
\hline
\end{tabular}

${ }^{\star} \mathrm{p}<.05 .{ }^{* \star} \mathrm{p}<.01$. $\dagger$ For vaginal sex only. Note: $\mathrm{p}$ values are for McNemar's test, which considers discordant responses only. 
stance use, sexual behavior and infection.

The general lack of significant differences between main and side partners may have a couple of explanations. One is our small sample size. Had our sample been larger, there may have been enough statistical power to detect a difference between main and side partners. A sample of 1,150 youth showed significantly more consistent condom use with "well-known partners" (who appear to be conceptually similar to our side partners) than with main partners. ${ }^{20}$

Another possible explanation is that main and side partners do not differ; a side partner may best be understood as a second main partner. The extent of concordance in behaviors with main and side partners found in our analysis would support this explanation.

Our results indicate little difference between main and side partners, and some differences between main and casual partners. Although we did not analyze side vs. casual partners, these results imply a difference. Future research based on larger samples should pursue the similarity in behavior and relationship characteristics of main and side partners, and further examine side partners as a separate entity from casual partners. Simply categorizing partners as main and other may introduce some misclassification, making it hard to identify some statistical associations.

The risk of STD acquisition appears to be higher with main partners than with casual partners. To date, safer sex messages may have been more effective in communicating the risks associated with casual partners than those associated with main partners. Our findings emphasize the need to educate men and women who have several partners that they are at risk even, or perhaps especially, in their primary relationships, and that using condoms in these relationships can be in accord with feelings of love and intimacy while at the same time providing protection against STDs. Pregnancy and disease prevention should be discussed at the same time, to ensure that clients understand how to prevent both effectively or how to reduce risk of disease when planning a pregnancy. This issue is of particular concern, since $44 \%$ of men and $76 \%$ of women in this study reported that their main partner had other partners.

The differences we found between the ways men and women behave with their main and their casual partners underscore the need for specificity in asking about sexual behaviors. Health care providers obtaining a sexual history or doing a risk assessment should ask clients whether they have multiple partners and, if so, about specific sexual and other risk behaviors related to HIV for each partner. From a disease prevention perspective, for individuals with multiple partners, it is important to disaggregate risk into behaviors that occur with each partner, as protective behaviors must occur with all partners in order to effectively prevent infection. Consequently, differential prevention messages should be delivered about the use of condoms and other contraceptives to prevent disease and pregnancy according to the individual's risks and reproductive intentions.

The use of individual-level analyses in this analysis identified some behaviors that are partner-specific and others that appear to be common with all partner types. These findings underscore the importance of conducting individual-level analyses, using appropriate statistical methods. Because persons with multiple partner types may behave differently than those with one partner type, such analyses will indicate whether group-level differences do, as we found, hold at the individual level.

\section{References}

1. Centers for Disease Control and Prevention (CDC), HIV/AIDS Surveillance Report, 1996, 8(2):11-12.

2. Update: barrier protection against HIV infection and other sexually transmitted diseases, Morbidity and Mortality Weekly Report, 1993, 42(30):589-591 \& 597.

3. CDC, Sexually Transmitted Disease Surveillance, 1995, Atlanta: CDC, 1996, pp. 33-53.

4. Becker MH and Joseph JG, AIDS and behavioral change to reduce risk: a review, American Journal of Public Health, 1988, 78(4):394-410.

5. Plichta SB et al., Partner-specific condom use among adolescent women clients of a family planning clinic, Journal of Adolescent Health, 1992, 13(6):506-511.

6. Worth $\mathrm{D}$, Sexual decision-making and AIDS: why condom promotion among vulnerable women is likely to fail, Studies in Family Planning, 1989, 20(6, pt. 1):297-307.
7. Thomas JC, Kulik AL and Schoenbach VJ, Syphilis in the South: rural rates surpass urban rates in North Carolina, American Journal of Public Health, 1995, 85(8, pt. 1):1119-1122; and Thomas, JC et al., Rural gonorrhea in the southeastern United States: a neglected epidemic? American Journal of Epidemiology, 1996, 143(3):269-277.

8. Distribution of STD clinic patients along a stages-ofbehavioral-change continuum-selected sites, 1993, Morbidity and Mortality Weekly Report, 1993, 42(45):880-883; Maxwell AE, Bastani R and Yan KX, AIDS risk behaviors and correlates in teenagers attending sexually transmitted diseases clinics in Los Angeles, Genitourinary Medicine, 1995, 71(2):82-87; and Weinstock HS et al., Factors associated with condom use in a high-risk heterosexual population, Sexually Transmitted Diseases, 1993, 20(1):14-20.

9. Soskolne $\mathrm{V}$ et al., Condom use with regular and casual partners among women attending family planning clinics, Family Planning Perspectives, 1991, 23(5):222-225.

10. Galavotti $C$ et al., Validation of measures of condom and other contraceptive use among women at high risk for HIV infection and unintended pregnancy, Health Psychology, 1995, 14(6):570-578.

11. Grimley DM et al., Contraceptive and condom use adoption and maintenance: a stage paradigm approach, Health Education Quarterly, 1995, 22(1):20-35.

12. Dolcini MM et al., Demographic characteristics of heterosexuals with multiple partners: the National AIDS Behavioral Survey, Family Planning Perspectives, 1993, 25(5):208-214; and Leigh BC, Temple MT and Trocki KF, The sexual behavior of US adults: results from a national survey, American Journal of Public Health, 1993, 80(10):1400-1408.

13. Messiah A, Pelletier A and the ACSF Group, Partner-specific sexual practices among heterosexual men and women with multiple partners: results from the French National Survey, ACSF, Archives of Sexual Behavior, 1996, 25(3):233-247.

14. Catania J et al., Risk factors for HIV and other sexually transmitted diseases and prevention practices among US heterosexual adults: changes from 1990 to 1992, American Journal of Public Health, 1995, 85(11):1492-1499.

15. Norris AE et al., Heterosexual experiences and partnerships of urban, low-income African-American and Hispanic youth, Journal of Acquired Immune Deficiency Syndromes and Human Retrovirology, 1996, 11(3):288-300.

16. van der Straten A et al., Sexual risk behavior with primary and non-primary partners among heterosexuals in HIV-serodiscordant couples, paper presented at the International Conference on AIDS, Vancouver, Canada, July 7-12, 1996.

17. Plichta SB et al., 1992, op. cit. (see reference 5).

18. Marx R et al., Crack, sex, and STD, Sexually Transmitted Diseases, 1991, 18(2):92-101.

19. Martin JL and Hasin DS, Drinking, alcoholism, and sexual behavior in a cohort of gay men, in: Fisher DG, ed., AIDS and Alcohol/Drug Abuse: Psychosocial Research, New York: Haworth Press, 1991, p. 50.

20. Norris AE et al., 1996, op. cit. (see reference 15). 